\title{
Learning difficulties in children attending a special clinic at the Lady Ridgeway Hospital
}

\author{
W G D T D Wijeratne ${ }^{1}$, N W N Y Wijesekera ${ }^{1}$, R T Wijesinghe ${ }^{1}$, S H Kariyawasam ${ }^{2}$
}

Sri Lanka Journal of Child Health, 2003; 32: 96-104

(Key words: Learning difficulties, children)

\begin{abstract}
Objectives To identify presentations of children with learning difficulties attending Child Psychiatry and Guidance Clinic (CPGC) at Lady Ridgeway Hospital (LRH) and describe their socio-demographic characteristics, health-seeking behaviour and modes of referral.
\end{abstract}

Design Descriptive cross sectional study.

Method Children diagnosed to have learning difficulties or attention deficit hyperactive disorder (ADHD), presenting to CPGC at LRH from 27 May to 10 June 2003, were included in study. Learning difficulties were diagnosed by a child psychiatrist using DSM IV criteria. A pre-tested, interviewer administered questionnaire (IAQ) was used to collect socio-demographic data, details on health-seeking behaviour and modes of referral from parents/ guardians. A checklist was used to identify presentations.

Results 52 children with learning difficulties were identified and all participated in study. Presentations were with difficulties in reading $(70 \%)$, mathematics $(60 \%)$, writing $(55 \%)$, communication $(45 \%)$ and motor skills (50\%). ADHD was found in $60 \%$ cases. $85 \%$ were males. Mean age was 9 years. $31 \%$ had deficit of schooling. 54\% were urban dwellers. Caretaker was mother in $83 \%$ cases. Monthly income was greater than 3000 rupees in $92 \%$ cases. $89 \%$ children were identified by mother or teacher. Initial visits were made to a specialist unit $(62 \%)$ or a primary health care service $(31 \%)$.

\section{Introduction}

For many children learning is a happy and enjoyable experience but some have learning difficulties. According to DSM IV, learning, communication and

\footnotetext{
${ }^{1}$ Medical student, ${ }^{2}$ Senior Lecturer, Dept. of Pharmacology, Faculty of Medicine, University of Colombo.
}

(Received on 15 October 2003) motor skills disorders are classified under learning difficulties ${ }^{1}$. Though technically not considered a learning difficulty there is a co-morbidity rate of 10 60\% between Attention Deficit Hyperactive Disorder (ADHD) and learning difficulties ${ }^{2}$. It is estimated that 5\% school children and 50\% children attending child psychiatry clinics in United States of America (USA) have learning difficulties ${ }^{1}$. Sri Lankan prevalence for learning difficulties is not known but a study done by Kariyawasam et $\mathrm{al}^{3}$ found ADHD a significant problem in Sri Lanka.

By definition, learning difficulties exclude sociocultural factors that may affect child's learning ${ }^{4,5}$ However, poor socio-economic conditions are associated with malnutrition, limited prenatal and postnatal care, exposure to teratogens and maternal substance abuse which can lead to subtle neuropsychiatric disturbances giving rise to learning difficulties $^{6}$. Recent studies have shown that characteristics of child's immediate environment have an impact on his maturation and indirectly on learning as well ${ }^{7}$.

Recognition of the true characteristics of children with learning difficulties will lead parents and teachers to deal with them in a sympathetic yet effective manner ${ }^{7}$. DSM IV gives criteria for diagnosis of learning difficulties ${ }^{1}$ Subtle characteristics and their predictability as high, moderate and weak have been described which may help identify children with learning difficulties ${ }^{8,9}$. Unfortunately these children are often not identified till late. This delays benefits of interventional care. If doctors, teachers and parents are vigilant about these characteristics, they can be identified early.

Early intervention for learning difficulties is warranted for maximal potential outcome in these children $^{7}$. In USA, where there is a proper network of interventional centres, different modalities of intervention have been studied and compared ${ }^{10}$. It has been recommended to establish regional healthcare teams that can liaise with the schools for the child neuropsychiatric disorders such as ADHD and other learning disabilities under the supervision of a paediatrician/psychiatrist ${ }^{3}$. 


\section{Method}

A descriptive cross sectional study was carried out in the Child Psychiatry and Guidance Clinic (CPGC) at Lady Ridgeway Hospital (LRH) from 27 May to 10 June 2003. Study population consisted of all children, 514 years old, diagnosed by a child psychiatrist as having a learning difficulty or ADHD using DSM IV criteria with diagnosis stated in child's clinic records. Children with mental retardation, autism, visual or hearing disabilities, confirmed by written records at CPGC, and those without documented evidence of learning difficulties were excluded from study,

CPGC functions twice weekly for 3 hours and about 20 children attend clinic each day. Considering feasibility of collecting data, a sample of 50 and a study period of 2 weeks was decided upon. An interviewer-administered questionnaire (IAQ) was used to assess sociodemographic characteristics, health seeking behaviour and modes of referral of children with learning difficulties. An interviewer-administered checklist (CL) was used to identify features at presentation. CL was devised using DSM IV criteria ${ }^{1}$, child psychiatry text books $^{6,11}$ and related research articles ${ }^{8,9}$. Both IAQ and CL were subjected to a focus group discussion of parents and teachers from Centre for Individuals with Learning Difficulties at Narahenpita. To minimize errors, IAQ comprised both open and close-ended questions, in a simple format, relating to a sequence approach to events, to improve recall. IAQ and CL were also subjected to the retranslation technique to improve validity and assess degree of agreement. To minimise errors in data transfer, a code column was included in IAQ. Medical students involved in study administered questionnaire after a training session. Both IAQ and CL were validated by a pretest on a sample of 5 children each, with and without learning difficulties, in ward 4, LRH and Centre for Individuals with Learning Difficulties. Reading and writing were not assessed in children below 7 years of age and mathematics in children below 8 years of age, as these are the current international recommendations ${ }^{1}$.

In collecting data, CPGC records were checked in all children presenting to clinic during study period, in the order of registration. After selecting children who met required criteria, an information leaflet on the study and its potential benefits was given to each parent/ guardian and informed verbal consent obtained. To maintain privacy, IAQ was individually administered in cubicles of the clinic room. Data was entered using Microsoft Excel Spread Sheet. Chi-square test was used to study significance of difference in socio-demographic characteristics.

\section{Results}

1. Presentations of children with learning difficulties

\section{a. Reading}

As cut-off age to assess reading is 7 years, it was assessed in only 41 children. Frequency of presentation with reading difficulties is shown in table 1

Table 1

Frequency of presentation with reading difficulties

\begin{tabular}{|l|l|}
\hline \multicolumn{1}{|c|}{ Presentation } & \multicolumn{1}{|c|}{ No (\%) } \\
\hline Has difficulty in spelling & $32(78.0)$ \\
\hline $\begin{array}{l}\text { Reading matter is changed by } \\
\text { omitting, adding, distorting }\end{array}$ & $32(78.0)$ \\
\hline Cannot read a paragraph by 8 years & $22(78.6)$ \\
\hline $\begin{array}{l}\text { Have problems in recognizing and } \\
\text { reading written words and letters }\end{array}$ & $29(70.7)$ \\
\hline $\begin{array}{l}\text { Uses different pronunciation for } \\
\text { letters B-P }\end{array}$ & $24(585)$ \\
\hline
\end{tabular}

$20(49 \%)$ children had all 5 presentations, $6(15 \%)$ had 4 presentations, $7(17 \%)$ had 3 presentations. $2(5 \%)$ had 2 presentations and $2(5 \%)$ had 1 presentation $4(10 \%)$ children had no reading difficulties.

\section{b. Mathematics}

As cut-off age to assess mathematics is 8 years, it was assessed in only 28 children. Frequency of presentation with mathematic difficulties is shown in table 2 .

Table 2

Frequency of presentation with difficulties in mathematics

\begin{tabular}{|l|c|}
\hline \multicolumn{1}{|c|}{ Presentation } & No (\%) \\
\hline $\begin{array}{l}\text { Cannot follow a sequence of } 3 \\
\text { mathematic steps }\end{array}$ & $21(750)$ \\
\hline $\begin{array}{l}\text { Have difficulties in putting written } \\
\text { problems into numbers }\end{array}$ & $18(64.3)$ \\
\hline $\begin{array}{l}\text { Poor in following multiplication } \\
\text { table by 10 years }\end{array}$ & $17(85.0)$ \\
\hline $\begin{array}{l}\text { Cannot remember to add/carry } \\
\text { forward }\end{array}$ & $16(57.1)$ \\
\hline Has problems in counting objects & $15(53.6)$ \\
\hline $\begin{array}{l}\text { See thing in wrong sequence; } 135 \\
\text { as } 153\end{array}$ & $14(50.0)$ \\
\hline $\begin{array}{l}\text { Has problems in recognizing } \\
\text { numerical symbols }\end{array}$ & $12(42.9)$ \\
\hline
\end{tabular}

Seven $(25 \%)$ children had all 7 presentations, $2(7 \%)$ had 6 presentations, $7(25 \%)$ had 5 presentations, 2 (7\%) had 4 presentations, 2 (7\%) had 3 presentations, 1 (4\%) had 2 presentations and $1(4 \%)$ had 1 presentation. $6(21 \%)$ children had no mathematic difficulties. 


\section{c. Writing}

As cut-off age to assess writing is 7 years it was assessed in only 41 children. Frequency of presentations with writing difficulties is shown in table 3 .

Table 3

Frequency of presentation with difficulties in writing

\begin{tabular}{|l|c|}
\hline \multicolumn{1}{|c|}{ Presentation } & No. (\%) \\
\hline $\begin{array}{l}\text { Difficulty in composing written } \\
\text { words }\end{array}$ & $27(65.9)$ \\
\hline Capital simple mixture & $24(58.5)$ \\
\hline Letter substitution B for P, U for N & $23(56.1)$ \\
\hline $\begin{array}{l}\text { Spelling errors-Not even appropriate } \\
\text { sound }\end{array}$ & $21(51.2)$ \\
\hline Letter sequence mixed eht for the & $19(46.3)$ \\
\hline
\end{tabular}

Fourteen (34\%) had all 5 presentations, $2(5 \%)$ had 4 presentations, $4(10 \%)$ had 3 presentations, $9(22 \%)$ had 2 presentations and $5(12 \%)$ had 1 presentation. $7(17 \%)$ children had no writing difficulties.

\section{d. Communication}

This was assessed in all 52 children. Frequency of presentations with communication difficulties is shown in table 4.

\section{Table 4}

Frequency of presentation with communication difficulties

\begin{tabular}{|l|c|}
\hline \multicolumn{1}{|c|}{ Presentation } & No. (\%) \\
\hline Receptive & $26(50.0)$ \\
\hline Appears to be deaf when spoken to & $26(50.0)$ \\
\hline $\begin{array}{l}\text { Gets disturbed when given instructions } \\
\text { for exercise }\end{array}$ & $23(44.2)$ \\
\hline $\begin{array}{l}\text { Difficulty in recognizing rhyming } \\
\text { words by age 4 }\end{array}$ & $29(55.8)$ \\
\hline Expressive & $23(44.2)$ \\
\hline $\begin{array}{l}\text { Eager to communicate but has } \\
\text { difficulty in finding the right word }\end{array}$ & $21(40.4)$ \\
\hline $\begin{array}{l}\text { By age 8, child only speaks short } \\
\text { phrases }\end{array}$ & $18(34.6)$ \\
\hline $\begin{array}{l}\text { Does not use grammar appropriate for } \\
\text { age }\end{array}$ & $25(48.1)$ \\
\hline $\begin{array}{l}\text { Difficulty in recalling common } \\
\text { information fast enough }\end{array}$ & $24(46.2)$ \\
\hline Phonological & $\begin{array}{l}\text { Poor articulation of later acquired } \\
\text { speech sound. R, SH, TH }\end{array}$ \\
\hline $\begin{array}{l}\text { Words sound like baby talk. Bu for } \\
\text { Blue, Wabbit for Rabbit }\end{array}$ & \\
\hline
\end{tabular}

\section{Receptive communication difficulties}

Thirteen (25\%) children had all 3 presentations, 22 $(42 \%)$ had 2 presentations and 13 (25\%) had 1 presentation. $4(8 \%)$ children had no receptive communication difficulties.

\section{Expressive communication difficulties}

Fourteen (27\%) children had all 4 presentations, 10 $(19 \%)$ had 3 presentations, $10(19 \%)$ had 2 presentations and $9(17 \%)$ had 1 presentation. $9(17 \%)$ children had no expressive communication difficulties.

\section{Phonological communication difficulties}

Twenty one (40\%) had 2 presentations and $15(29 \%)$ had 1 presentation. $16(31 \%)$ had no phonological communication difficulties.

\section{e. Motor skills}

This was assessed in all 52 children. Frequency of presentations with motor skills difficulties is shown in table 5.

\section{Table 5}

Frequency of presentations with motor skills difficulties

\begin{tabular}{|l|l|}
\hline \multicolumn{1}{|c|}{ Presentation } & No (\%) \\
\hline Clumpy and messy at work & $32(61.5)$ \\
\hline $\begin{array}{l}\text { Difficulty in tying shoelaces, } \\
\text { buttoning shirts by age 6 }\end{array}$ & $27(60.0)$ \\
\hline Has problems in model building & $19(36.5)$ \\
\hline $\begin{array}{l}\text { Has problems in playing games } \\
\text { with hand eye coordination - } \\
\text { Tennis, Badminton, Football as } \\
\text { opposed to swimming, running }\end{array}$ & $14(26.9)$ \\
\hline Handwriting poor & $34(65.4)$ \\
\hline Pressing too hard on the paper & $34(65.4)$ \\
\hline $\begin{array}{l}\text { Sharpening the pencil every } \\
\text { minute }\end{array}$ & $29(55.8)$ \\
\hline $\begin{array}{l}\text { Erasing what was written every } \\
\text { word or 2 }\end{array}$ & $21(40.4)$ \\
\hline $\begin{array}{l}\text { Difficulty in copying blackboard, } \\
\text { text or notebook }\end{array}$ & $14(26.9)$ \\
\hline $\begin{array}{l}\text { With palmar grasp and bended } \\
\text { wrists }\end{array}$ & \\
\hline
\end{tabular}

\section{Handwriting}

Five (10\%) had all 5 presentations, $10(19 \%)$ had 4 presentations, $18(35 \%)$ had 3 presentations, 9 (17\%) had 2 presentations and $6(11 \%)$ had 1 presentation. 4 $(8 \%)$ children had no handwriting difficulties.

\section{Other motor skills}

Six $(12 \%)$ had all 4 presentations, $8(15 \%)$ had 3 presentations, $17(33 \%)$ had 2 presentations and 11 (21\%) had 1 presentation. 10 (19\%) had no difficulties in other motor skills. 


\section{f. Attention deficit/Hyperactivity}

This was assessed in all 52 children. Frequency of presentations with attention deficit/ hyperactivity is shown in table 6

Table 6

Frequency of presentations with attention deficit/hyperactivity

\begin{tabular}{|c|c|}
\hline Presentation & No. (\%) \\
\hline \multicolumn{2}{|l|}{ Inattention } \\
\hline $\begin{array}{l}\text { Is easily distracted by extraneous } \\
\text { stimuli }\end{array}$ & $44(84.6)$ \\
\hline $\begin{array}{l}\text { Fails to give close attention to } \\
\text { details or makes careless } \\
\text { mistakes }\end{array}$ & $42(80.8)$ \\
\hline $\begin{array}{l}\text { Does not seem to listen when } \\
\text { spoken to directly }\end{array}$ & $41(78.8)$ \\
\hline $\begin{array}{l}\text { Has difficulty in sustaining } \\
\text { attention in task or play activities }\end{array}$ & $40(76.9)$ \\
\hline $\begin{array}{l}\text { Avoids, dislikes to engage in } \\
\text { tasks that require sustained } \\
\text { mental effort }\end{array}$ & $40(76.9)$ \\
\hline $\begin{array}{l}\text { Does not follow through } \\
\text { instructions and fails to finish } \\
\text { schoolwork }\end{array}$ & $39(75.0)$ \\
\hline Is forgetful in daily activities & $33(63.5)$ \\
\hline $\begin{array}{l}\text { Has difficulty in organizing } \\
\text { tasks and activities }\end{array}$ & $31(59.6)$ \\
\hline $\begin{array}{l}\text { Loses things needed for } \\
\text { activities }\end{array}$ & $31(59.6)$ \\
\hline \multicolumn{2}{|l|}{ Hyperactivity } \\
\hline $\begin{array}{l}\text { Leaves seat in situation in which } \\
\text { remaining seated is expected }\end{array}$ & $38(73.1)$ \\
\hline $\begin{array}{l}\text { Fidgets with hand or feet or } \\
\text { squirms in seat }\end{array}$ & $36(69.2)$ \\
\hline $\begin{array}{l}\text { Runs about or climbs } \\
\text { excessively in inappropriate } \\
\text { situations }\end{array}$ & $35(67.3)$ \\
\hline $\begin{array}{l}\text { Is "on the go" or acts as if driven } \\
\text { by motor }\end{array}$ & $28(53.8)$ \\
\hline $\begin{array}{l}\text { Has difficulty in engaging in } \\
\text { leisure activities quietly }\end{array}$ & $27(51.9)$ \\
\hline Talks excessively & $25(48.1)$ \\
\hline \multicolumn{2}{|l|}{ Impulsivity } \\
\hline Interrupts or intrudes on others & $22(42.3)$ \\
\hline Has difficulty in waiting for turn & $19(36.5)$ \\
\hline $\begin{array}{l}\text { Blurts out answers before } \\
\text { questions have been completed }\end{array}$ & $15(30.8)$ \\
\hline
\end{tabular}

\section{Inattention}

Ten (19\%) had all 9 presentations, 10 (19\%) had 8 presentations, $13(25 \%)$ had 7 presentations, $8(15 \%)$ had 6 presentations, $4(8 \%)$ had 4 presentations, 3
(6\%) had 3 presentations and $3(6 \%)$ had 2 presentations. $1(2 \%)$ child had no inattention.

\section{Hyperactivity}

Fourteen (27\%) had all 6 presentations, $14(27 \%)$ had 5 presentations, $8(15 \%)$ had 4 presentations, $1(2 \%)$ had 3 presentations, $4(8 \%)$ had 2 presentations and 4 $(8 \%)$ had 1 presentation. 7 (14\%) children had no hyperactivity.

\section{Impulsivity}

Fourteen (27\%) had all 3 presentations, $10(19 \%)$ had 2 presentations and $14(27 \%)$ had 1 presentation. 14 $(27 \%)$ children had no impulsivity.

\section{Socio-demographic characteristics}

\section{a. Gender}

Forty four $(85 \%)$ children were male and $8(15 \%)$ were female.

b. Age

Distribution of age is shown in table 7

Table 7

Distribution of age

\begin{tabular}{|c|c|}
\hline Age Group & No (\%) \\
\hline $4 \mathrm{yr} 6 \mathrm{~m}-7 \mathrm{yr} 5 \mathrm{~m}$ & $18(35)$ \\
\hline $7 \mathrm{yr} 6 \mathrm{~m}-10 \mathrm{yr} 5 \mathrm{~m}$ & $21(40)$ \\
\hline $10 \mathrm{yr} 6 \mathrm{~m}-13 \mathrm{yr} 5 \mathrm{~m}$ & $13(25)$ \\
\hline Total & $\mathbf{5 2 ( 1 0 0 )}$ \\
\hline
\end{tabular}

The mean age was 9 years (SD $2 \mathrm{yr} 4 \mathrm{~m}$ ).

\section{c. Deficit of schooling}

Deficit of schooling among children is shown in table 8 .

Table 8

Deficit of schooling among children

\begin{tabular}{|l|c|}
\hline \multicolumn{1}{|c|}{ Deficit } & No. (\%) \\
\hline No deficit & $36(69)$ \\
\hline 1year & $05(10)$ \\
\hline$>$ 1year & $02(04)$ \\
\hline Special schools & $09(17)$ \\
\hline Total & $\mathbf{5 2 ( 1 0 0 )}$ \\
\hline
\end{tabular}

\section{d District}

Distribution district-wise is shown in table 9. 
Table 9

Distribution district-wise

\begin{tabular}{|l|c|}
\hline \multicolumn{1}{|c|}{ District } & No. (\%) \\
\hline Colombo & $23(44)$ \\
\hline Gampaha & $16(31)$ \\
\hline Kalutara & $04(08)$ \\
\hline Kurunegala & $03(06)$ \\
\hline Kegalle & $03(06)$ \\
\hline Negombo & $01(02)$ \\
\hline Matara & $01(02)$ \\
\hline Ratnapura & $01(02)$ \\
\hline Total & $\mathbf{5 2 ( 1 0 0 )}$ \\
\hline
\end{tabular}

\section{e. Urban/Rural}

Distribution according to residence (urban/rural) shown in table 10 .

Table 10

Distribution according to dwelling

\begin{tabular}{|l|c|}
\hline \multicolumn{1}{|c|}{ Dwelling } & No. (\%) \\
\hline Urban & $28(54)$ \\
\hline Rural & $24(46)$ \\
\hline Total & $\mathbf{5 2 ( 1 0 0 )}$ \\
\hline
\end{tabular}

\section{f. Care-taker}

Type of care-taker is shown in table 11.

Table 11

Type of care-taker

\begin{tabular}{|l|c|}
\hline \multicolumn{1}{|c|}{ Care-taker } & No. (\%) \\
\hline Mother & $43(82.7)$ \\
\hline Father & $00(0.0)$ \\
\hline Grandmother & $05(9.6)$ \\
\hline Servant & $02(3.8)$ \\
\hline Uncle & $01(1.9)$ \\
\hline Orphanage & $01(1.9)$ \\
\hline Total & $\mathbf{5 2 ( 1 0 0 )}$ \\
\hline
\end{tabular}

\section{g. Care-taker's education level}

Care-taker's education level is shown in table 12.

Table 12

Caretaker's education level

\begin{tabular}{|l|c|}
\hline Maximum education & No. (\%) \\
\hline No schooling & $01(01.9)$ \\
\hline Primary education & $15(28.8)$ \\
\hline Up to ordinary level & $20(38.5)$ \\
\hline Up to advanced level & $13(25.0)$ \\
\hline Tertiary professional & $03(05.8)$ \\
\hline Total & $\mathbf{5 2 ( 1 0 0 )}$ \\
\hline
\end{tabular}

\section{h. Job status of the family}

Number of family members who are working is shown in table 13.

Table 13

No. of family members who are working

\begin{tabular}{|l|c|}
\hline \multicolumn{1}{|c|}{ People working } & No. (\%) \\
\hline Father only & $32(61.5)$ \\
\hline Father \& Mother & $16(30.8)$ \\
\hline Guardian only & $03(05.8)$ \\
\hline Mother only & $01(01.9)$ \\
\hline Total & $\mathbf{5 2 ( 1 0 0 )}$ \\
\hline
\end{tabular}

Type of job of family member is shown in table 14 .

Table 14

Type of job of family member

\begin{tabular}{|l|c|}
\hline \multicolumn{1}{|c|}{ Type of job } & No. (\%) \\
\hline Professional, technical & $04(05.9)$ \\
\hline Executive \& management & $08(11.8)$ \\
\hline Clerical & $08(11.8)$ \\
\hline Sales personnel & $12(17.6)$ \\
\hline Service providers & $19(27.9)$ \\
\hline Agriculture \& animal husbandry & $03(04.4)$ \\
\hline Production, transport \& labour & $10(14.7)$ \\
\hline Other & $04(05.9)$ \\
\hline Total & $\mathbf{6 8 ( 1 0 0 )}$ \\
\hline
\end{tabular}

i. Monthly income

Income level is shown in table 15.

Table 15

Level of income

\begin{tabular}{|l|c|}
\hline \multicolumn{1}{|c|}{ Income } & No. $(\%)$ \\
\hline$<3,000$ & $04(07.7)$ \\
\hline $3000-10,000$ & $30(57.7)$ \\
\hline$>10,000$ & $18(34.6)$ \\
\hline Total & $\mathbf{5 2 ( 1 0 0 )}$ \\
\hline
\end{tabular}

\section{j. Family Size}

Number of people living in household is shown in table 16.

Table 16

No. of people living in the household

\begin{tabular}{|c|c|}
\hline Family Size & No. (\%) \\
\hline Three & $14(26.9)$ \\
\hline Four & $20(38.5)$ \\
\hline Five & $12(23.1)$ \\
\hline Six & $02(03.8)$ \\
\hline$>\operatorname{six}$ & $04(07.7)$ \\
\hline Total & $52(100)$ \\
\hline
\end{tabular}




\section{k.. Siblings}

Number of siblings of index child is shown in table 17.

Table 17

Number of siblings

\begin{tabular}{|l|c|}
\hline \multicolumn{1}{|c|}{ Siblings } & No. (\%) \\
\hline None & $22(42.3)$ \\
\hline One & $20(38.5)$ \\
\hline Two & $09(17.3)$ \\
\hline Three & $01(01.9)$ \\
\hline Total & $\mathbf{5 2 ( 1 0 0 )}$ \\
\hline
\end{tabular}

No deficit of schooling was seen in siblings.

\section{Race}

Distribution by race is shown in table 18 .

Table 18

Distribution according to race

\begin{tabular}{|l|c|}
\hline \multicolumn{1}{|c|}{ Race } & No. (\%) \\
\hline Sinhala & $46(88.4)$ \\
\hline Tamil & $03(05.8)$ \\
\hline Muslim & $03(05.8)$ \\
\hline Total & $\mathbf{5 2 ( 1 0 0 )}$ \\
\hline
\end{tabular}

\section{m. Religion}

Distribution by religion is shown in table 19.

Table 19

Distribution according to religion

\begin{tabular}{|l|c|}
\hline \multicolumn{1}{|c|}{ Religion } & No. (\%) \\
\hline Buddhist & $40(76.9)$ \\
\hline Hindhu & $00(00.0)$ \\
\hline Christian & $09(17.3)$ \\
\hline Islam & $03(05.8)$ \\
\hline Total & $\mathbf{5 2 ( 1 0 0 )}$ \\
\hline
\end{tabular}

\section{Health seeking behaviour}

\section{a. Who noticed the difference?}

Person who identified is shown in table 20.

Table 20

Person who noticed the difference in the child

\begin{tabular}{|l|c|}
\hline \multicolumn{1}{|c|}{ Person } & No. (\%) \\
\hline Mother & $33(63.5)$ \\
\hline Teacher & $13(25.0)$ \\
\hline Father & $00(00.0)$ \\
\hline Other & $06(11.5)$ \\
\hline Total & $\mathbf{5 2 ( 1 0 0 )}$ \\
\hline
\end{tabular}

\section{b. Age of identification}

Age of identification is given in table 21
Table 21

Age of identification

\begin{tabular}{|c|c|}
\hline $\begin{array}{c}\text { Age of identification } \\
(y r s)\end{array}$ & No. (\%) \\
\hline 0.3 & $02(03.8)$ \\
\hline 0.5 & $01(01.9)$ \\
\hline 1.0 & $01(01.9)$ \\
\hline 1.5 & $05(09.6)$ \\
\hline 2.0 & $03(05.8)$ \\
\hline 2.5 & $01(01.9)$ \\
\hline 3.0 & $05(09.6)$ \\
\hline 3.5 & $01(01.9)$ \\
\hline 4.0 & $05(09.6)$ \\
\hline 5.0 & $08(1-5.4)$ \\
\hline 5.5 & $02(03.8)$ \\
\hline 6.0 & $10(19.2)$ \\
\hline 7.0 & $03(05.8)$ \\
\hline 8.0 & $01(01.9)$ \\
\hline 9.0 & $01(01.9)$ \\
\hline 10.0 & $03(05.8)$ \\
\hline Total & $\mathbf{5 2 ( 1 0 0 )}$ \\
\hline
\end{tabular}

The mean age of identification was 4.53 years.

\section{c. Time period taken for first intervention}

Time taken for 1 st intervention is shown in table 22 .

Table 22

Time taken for first intervention

\begin{tabular}{|c|c|}
\hline Time in months & No. (\%) \\
\hline$<3$ & $26(50.0)$ \\
\hline $3-6$ & $02(03.8)$ \\
\hline $7-12$ & $00(00.0)$ \\
\hline$>12$ & $24(46.2)$ \\
\hline Total & $\mathbf{5 2 ( 1 0 0 )}$ \\
\hline
\end{tabular}

\section{d Interventions}

Reasons for delay in intervention are shown in table 23.

Table 23

Reasons for delay in intervention

\begin{tabular}{|l|c|}
\hline \multicolumn{1}{|c|}{ Reason } & No. (\%) \\
\hline No delay & $24(46.2)$ \\
\hline Thought it was normal & $07(13.5)$ \\
\hline Only gradually progressed & $06(11.5)$ \\
\hline Thought will reduce with time & $04(07.7)$ \\
\hline Other illnesses & $04(07.7)$ \\
\hline Thought it was bad time & $02(03.8)$ \\
\hline Did not recognize & $02(03.8)$ \\
\hline Unavoidable circumstances & $02(03.8)$ \\
\hline Family history & $01(01.9)$ \\
\hline Total & $\mathbf{5 2}(\mathbf{1 0 0})$ \\
\hline
\end{tabular}

First intervention is shown in table 24. 
Table 24

First intervention

\begin{tabular}{|l|c|}
\hline \multicolumn{1}{|c|}{ Action } & No. (\%) \\
\hline $\begin{array}{l}\text { Informing someone } \\
\text { responsible }\end{array}$ & $04(07.7)$ \\
\hline $\begin{array}{l}\text { Went to primary health care } \\
\text { service }\end{array}$ & $16(30.8)$ \\
\hline Went to specialist unit & $32(61.5)$ \\
\hline Total & $\mathbf{5 2 ( 1 0 0 )}$ \\
\hline
\end{tabular}

Health personnel involved in intervention are shown in table 25 .

Table 25

Health personnel involved in interventions

\begin{tabular}{|l|c|}
\hline \multicolumn{1}{|c|}{ Person } & No. (\%) \\
\hline General Practitioner & $09(17.3)$ \\
\hline Psychiatrist & $36(69.2)$ \\
\hline Paediatrician & $31(59.6)$ \\
\hline Speech therapist & $05(09.6)$ \\
\hline Other & $03(05.8)$ \\
\hline Total & $\mathbf{5 2 ( 1 0 0 )}$ \\
\hline
\end{tabular}

The number of steps taken is shown in table 26 .

Table 26

Number of steps taken

\begin{tabular}{|c|c|}
\hline Number of steps & No. (\%) \\
\hline 1 & $06(11.54)$ \\
\hline 2 & $21(40.38)$ \\
\hline 3 & $22(42.31)$ \\
\hline 4 & $03(05.77)$ \\
\hline
\end{tabular}

Type of intervention is shown in table 27.

Table 27

Type of intervention

\begin{tabular}{|l|c|}
\hline \multicolumn{1}{|c|}{ Type of intervention } & No. (\%) \\
\hline $\begin{array}{l}\text { LRH learning difficulty } \\
\text { clinic }\end{array}$ & $52(100)$ \\
\hline Paediatrician & $20(38.5)$ \\
\hline LRH clinic & $18(34.6)$ \\
\hline $\begin{array}{l}\text { Psychiatrist (excl. } \\
\text { clinic) }\end{array}$ & $09(17.3)$ \\
\hline Local hospital & $08(15.4)$ \\
\hline General Practitioner & $07(13.5)$ \\
\hline Special schools & $06(11.5)$ \\
\hline Other official/institute & $06(11.5)$ \\
\hline
\end{tabular}

The order and types of intervention in sequence are shown in table 28 .
Table 28

Order and types of interventions taken in sequence

Type of intervention

Order of steps taken

$\begin{array}{lcccc} & 1 \text { st } & \text { 2nd } & \text { 3rd } & \text { 4th } \\ \text { Local hospital } & 08 & 00 & 00 & 00 \\ \text { Paediatrician } & 11 & 08 & 01 & 00 \\ \text { LRH clinic } & 11 & 07 & 00 & 00 \\ \text { LRH learning } & & & & \\ \text { difficulty clinic } & 06 & 21 & 23 & 02 \\ \text { Special schools } & 04 & 01 & 00 & 01 \\ \text { General practitioner } & 05 & 02 & 00 & 00 \\ \text { Psychiatrist } & 05 & 03 & 01 & 00 \\ \text { Other official/institute } & 02 & 04 & 00 & 00\end{array}$

Non-western interventions occurred in $25(48 \%)$ instances. Ayurveda was involved in $6(24 \%)$ and religious/spiritual interventions in 19 (76\%).

Period of intervention at CPGC is shown in table 29.

Table 29

Period of intervention at CPGC

\begin{tabular}{|c|c|}
\hline $\begin{array}{c}\text { Period of intervention in } \\
\text { months }\end{array}$ & No (\%) \\
\hline $00-03$ & $27(51.9)$ \\
\hline $04-06$ & $13(25.0)$ \\
\hline $07-12$ & $09(17.3)$ \\
\hline $13-23$ & $00(00.0)$ \\
\hline 24 or $>$ & $03(05.8)$ \\
\hline Total & $\mathbf{5 2}(\mathbf{1 0 0})$ \\
\hline
\end{tabular}

Source of information regarding CPGH is shown in table 30 .

Table 30

Source of information regarding CPGC

\begin{tabular}{|l|c|}
\hline \multicolumn{1}{|c|}{ Person/Institution } & No. $(\%$ \\
\hline LRH OPD & $10(19.3)$ \\
\hline Paediatrician & $10(19.3)$ \\
\hline Teacher & $09(17.3)$ \\
\hline Another hospital & $08(15.4)$ \\
\hline Relation & $05(09.6)$ \\
\hline General Practitioner & $04(07.7)$ \\
\hline Paramedical staff & $02(03.8)$ \\
\hline Psychiatrist & $02(03.8)$ \\
\hline Special institute & $01(01.9)$ \\
\hline Television & $01(01.9)$ \\
\hline Total & $\mathbf{5 2 ( 1 0 0 )}$ \\
\hline
\end{tabular}


Association between socio-demographic characteristics and presentations are shown in table 31 .

Table 31

Association between socio-demographic characteristics and presentation

\begin{tabular}{|l|l|}
\hline \multicolumn{1}{|c|}{ Presentation vs Characteristic } & P value \\
\hline $\begin{array}{l}\text { Pressing too hard on paper when } \\
\text { writing vs Income of family }\end{array}$ & $\mathrm{P}>0.05$ \\
\hline $\begin{array}{l}\text { Pressing too hard on paper when } \\
\text { writing vs caretaker's education level }\end{array}$ & $\mathrm{P}>0.05$ \\
\hline $\begin{array}{l}\text { Difficulty in composing written } \\
\text { words vs caretaker's education level }\end{array}$ & $\mathrm{P}>0.05$ \\
\hline $\begin{array}{l}\text { Reading matter is changed by } \\
\text { omitting, adding, distorting vs } \\
\text { caretaker's education level }\end{array}$ & $\mathrm{P}<0.05$ \\
\hline $\begin{array}{l}\text { Eager to communicate but has } \\
\text { difficulty in finding right word vs } \\
\text { presence of siblings }\end{array}$ & $\mathrm{P}>0.05$ \\
\hline $\begin{array}{l}\text { Is easily distracted by extraneous } \\
\text { stimuli vs age }\end{array}$ & $\mathrm{P}>0.05$ \\
\hline
\end{tabular}

\section{Discussion}

Reading difficulties were common presentations in children with learning difficulties in our study occurring in over $70 \%$ cases. Our results are compatible with those of Scarborough ${ }^{8}$. Mathematics is considered a good way of assessing learning difficulties as it objectively defines cut offs and is easily measurable ${ }^{1}$. In our study around $60 \%$ children presented with difficulties in mathematics. About 55\% children presented with difficulties in writing. Difficulties in communication were presenting features in about $45 \%$ children. Around $50 \%$ presented with difficulties in motor skills. Attention deficit and hyperactivity were found in about $60 \%$ children in our study.

Many learning difficulties including ADHD have male preponderance $^{1}$ and the male to female ratio for ADHD in Sri Lanka is 3.6:13. In our study $85 \%$ children were male. The mean age in the study sample was 9 years. $31 \%$ children had some deficits in schooling. $75 \%$ of the children were from the Colombo and Gampaha districts, a not unexpected finding. 54\% were urban dwellers. The mother was the care-taker in $83 \%$ cases. In $98 \%$ cases the care-taker had at least a primary education and in $67 \%$ instances had done their ordinary level examination. This finding is encouraging as strategies for information are delivered through the care-taker whose education level is important for the receptivity of such information. $92 \%$ of the families earned more than 3000 rupees a month. In $89 \%$ cases the family size was 5 or less and in $81 \%$ instances the sibling number was either one or none.
Association between socio-demographic characteristics and presentations was found to be significant only between a characteristic of reading (where words were added, omitted or distorted while reading) and the caretaker's education level.

In $89 \%$ cases the mother or teacher were responsible for identification of the child with the presentation disclosed. In a local study in $74 \%$ children the problems had been detected by parents or relatives ${ }^{13}$. Mean age of identification was 4.5 years. The time taken for the first intervention showed 2 peaks $50 \%$ taking less than 3 months and $46 \%$ taking more than a year. The first medically related action taken was to go to a specialist unit in $62 \%$ cases and the primary health care service in $31 \%$ instances. The psychiatrist (69\%) and the paediatrician $(60 \%)$ were the main people involved in the interventions. Of those who sought non-western interventions only $24 \%$ sought Ayurveda treatment before coming for medical interventions. Of those who sought treatment from CPGC only $23 \%$ attended the clinic for more than 6 months, probably implying good progress within a short time with the interventions provided at the CPGC although non-compliance with the passage of tine cannot be excluded. Similar levels of satisfaction have been noted in a local study ${ }^{14}$ where 165 new referrals were recruited of whom $66 \%$ expressed satisfaction with the interventions provided.

The general public, with special emphasis on primary school teachers, should be educated on the common presentations of learning difficulties. Proper education for identification and referral should be given to parents. First contact care health providers, too, need education on the presenting features and the availability of interventional centres.

\section{Limitations of study}

- Ideal study population would have been new enrolments to clinic. However, sample size would then be significantly reduced.

- Due to time lapse between presentation and date of interview, information gathered would carry a recall bias.

- Ideally study should have been conducted in clinics of an array of consultants to represent true population. We restricted our study to a specific clinic to ensure uniform assessment.

- A larger study sample, though ideal, was not possible due to time constraints. 


\section{Acknowledgements}

We thank Professor Dulitha Fernando, Head of Department of Community Medicine and all staff members, Professor Rohini Seneviratne, Dr. Hemamali Perera and other members of the Department of Psychiatry, Dr Charukshi Arambepola and Dr Ferdinando, Mrs Thezween Kariyawasam, Mrs T Samaraweera and all other teachers and parents of the Association for Individuals with Learning Difficulties, staff of the faculty library, Director and Librarian of "Sahanaya", non-academic staff of Faculty of Medicine, Colombo and all the parents/guardians of children with learning difficulties for their invaluable help.

\section{References}

1. American Psychiatric Association. Diagnostic and statistical manual of mental disorders. 4th ed. Washington DC; American Psychiatrists Association, 1994.

2. Beitchman J H, Young A R. Learning disorders with special emphasis on reading disorders: review of past 10 years. Journal of American Academy of Child and Adolescent Psychiatry 1997; 36: 102032.

3. Kariyawasam S H. Koralagama A, Jayawardane P. Karunathilake B, Perera V, Perera H. A descriptive study of attention deficit hyperactive disorder (ADHD) at Lady Ridgeway Hospital for Children, Colombo. Sri Lanka Journal of Child Health 2002; 31: 109-14.

4. Kavale K, Forness S. The nature of learning disabilities Hillsdale, NJ: Erlbaum, 1995.

5. Lyon G R. Learning disabilities. Future Child 1996: 6: 54-76.

6. Hallahan D P, Kauffman J M, Lloyd J W. Introduction to learning disabilities. Boston; Allyn and Bacon, 1996.
7. Theodore D Wachs. The enhancement of competence in the healthy child. Annales Nestle 2001; 59: 121-9.

8. Scarborough H S. Early identification of children at risk for reading disabilities; phonological awareness and some other promising predictors. In: Shapiro B K, Capture A J. editors. Specific reading disability: A view of the spectrum. Hillsdale. NJ; Erlbaum, 1998:77-81.

9. Fletcher J M, Foorman B R, Boudousquie A B et al. Assessment of reading and learning difficulties disabilities; a research based, intervention oriented approach. Journal of School Psychology, in press.

10. The National Institute of Child Health and Human Development- The report of the National Reading Panel. An evidence based assessment of the scientific literature on reading and its implications for reading instruction Bethesda MD; NICHD, 2000 .

11. J C Division, J M Neale. Abnormal Psychiatry. 6th edition. New York; John Whale and Sons Inc, 1994: 447-54.

12. Nikapota A D. School refusal - a review of some cases. Ceylon Journal of Child Health 1981; 1: 912.

13. Perera H, Perera R. User satisfaction with child psychiatry outpatient care: implications for practice. Ceylon Medical Journal 1998; 43:185-90.

14. Senanayake M P, Jayamaha D J M N R, De Silva T U N, Bahirathan S. Pathways taken by parents of disabled children. Ceylon Medical Journal 2000; 45(4): 179. 
